\title{
A Novel Approach for Eminent Data Hiding Based on Secret- Fragment-Visible Mosaic Images and Pixel Transformation for Secure Image Transmission
}

\author{
${ }^{1}$ Tummala Lakshmi Prasanthi, ${ }^{2}$ Shaik Mahaboob Basha and ${ }^{3}$ J. Srinivasulu \\ ${ }^{1}$ Post Graduate Student, Department of E.C.E., Priyadarshini College of \\ Engineering and Technology, Nellore, A.P., India \\ ${ }^{2}$ Professor and Head, Department of E.C.E, Priyadarshini College of Engineering \\ and Technology, Nellore, A.P., India \\ ${ }^{3}$ Assistant Professor, Department of E.C.E, Priyadarshini College of Engineering \\ and Technology, Nellore, A.P., India \\ 'santhi.ece.437@gmail.com, ${ }^{2}$ mohisin7@yahoo.co.in, ${ }^{3}$ jogee_s@yahoo.com
}

\begin{abstract}
Hiding the data in digital images has been area of interest in the digital image processing domain. Although so much work has been carried out in the literature to resolve the issues like increasing the data capacity, creating the secret image alike of target image but most of the works fails to meet the practical requirements. This paper presents an approach where mosaic image generation has done by dividing the secret image into fragments and transforming their respective colour characteristics into corresponding blocks of the target image. Usage of the Pixel colour transformations helps to yield the lossless recovered image based on the untransformed colour space values. Generation of the key plays an important role to recover the data from the secret image in lossless manner. Finally the same approach can be performed on videos also which helps to eliminate the flickering artifact to achieve the lossless data recovery in motion related videos. The experimental results shows good robust behavior against all incidental and accidental attacks and compare to the conventional algorithms performance evaluation has been increased in a significant way. The result of the proposed technique outperforms present techniques and the results are simulated using MATLAB.
\end{abstract}

Keywords: Image Transmission, Visual quality, Data hiding, Digital image

\section{Introduction}

Digital images have become very common in the present society needs, especially in the applications where security and privacy are essential features. The concept of image processing lead to the evolution of different image acquisition devices like still and video cameras, web cameras. Our work is to designate a novel technique for solving the safety data hiding procedure.

Currently, images from various sources are frequently utilized and transmitted through the internet for various applications, such as online personal photograph albums, confidential enterprise archives, document storage systems, medical imaging systems, and military image databases. These images usually contain private or confidential information so that they should be protected from leakages during transmissions.

This paper is organized as follows. The section 1 describes the introduction of the data hiding and necessity in the field. The section 2 named as Literature Survey as it explained various existing approaches and section 3 evaluated the proposed Methodology. The 
section 3.1 discussed the Mathematical basics for proposed work, The section 4 derived the Algorithmic flow and section 5 includes Simulation results. Finally the sections 6 and 7 described conclusion and future scope of the paper.

\section{Literature Survey}

Recently, many methods have been proposed for secure image transmission, for which two common approaches are image encryption and data hiding. Image encryption is a technique that makes use of the natural property of an image, such as high redundancy and strong spatial correlation, to get an encrypted image based on Shannon's confusion and diffusion properties [1-4, 13-15].In this, the encrypted image is a noise image so that no one can obtain the secret image from it unless he/she has the correct key. However, the encrypted image is a meaningless file, which cannot provide additional information before decryption and may arouse an attacker's attention during transmission due to its randomness in form. An alternative to avoid this problem is data hiding that hides a secret message into a cover image so that no one can realize the existence of the secret data, in which the data type of the secret message investigated in this paper, is an image. Existing data hiding methods mainly utilize the techniques of LSB sub situation, histogram shifting, difference expansion, prediction-error expansion, recursive histogram modification, and discrete cosine/wavelet transformations. However, in order to reduce the distortion of the resulting image, an upper bound for the distortion value is usually set on the payload of the cover image.

A discussion on this rate distortion issue can be found in. Thus, a main issue of the methods for hiding data in images is the difficulty to embed a large amount of message data into a single image. Specifically, if one wants to hide a secret image into a cover image with the same size, the secret image must be highly compressed in advance. For example, for a data hiding method with an embedding rate of 0.5 bits per pixel, a secret image with 8 bits per pixel must be compressed at a rate of at least $93.75 \%$ beforehand in order to be hidden into a cover image. But, for many applications, such as keeping or transmitting medical pictures, military images, legal documents, etc., that are valuable with no allowance of serious distortions, such data compression operations are usually impractical. Moreover, most image compression methods, such as JPEG compression, are not suitable for line drawings and textual graphics, in which sharp contrasts between adjacent pixels are often destructed to become noticeable artifacts. In this paper, a new technique for secure image transmission is proposed, which transforms a secret image into a meaningful mosaic image with the same size and looking like a preselected target image. The transformation process is controlled by a secret key, and only with the key can a person recover the secret image nearly lossless from the mosaic image. The proposed method is inspired by Lai and Tsai, in which a new type of computer art image, called secret-fragment-visible mosaic image, was proposed.

The mosaic image is the result of rearrangement of the fragments of a secret image in disguise of another image called the target image preselected from a database. But an obvious weakness of Lai and Tsai is the requirement of a large image database so that the generated mosaic image can be sufficiently similar to the selected target image. In this, the users are not allowed to select freely their interested image for use as the target image. Thus, the proposed method is to remove this weakness of the above method while keeping its merit, i.e., it is aimed to design a new method that can transform a secret image into a secret fragment visible mosaic image of the same size that has the visual appearance of any freely selected target image without the need of a database.

After a target image is selected arbitrarily, the given secret image is first decomposed into rectangular fragments called tile images, which then are fit into similar blocks in the target image, called target blocks, according to a similarity criterion based on colour variations. Next, the colour characteristic of each tile image is transformed to be that of 
the corresponding target block in the target image, resulting in a mosaic image which looks like the target image. Relevant schemes are also proposed to conduct nearly lossless recovery of the original secret image from the resulting mosaic image. The proposed method is new in that a meaningful mosaic image is created, in contrast with the image encryption method that only creates meaningless noise images. Also, the proposed method can transform a secret image into a disguising mosaic image without compression, while a data hiding method must hide a highly compressed version of the secret image into a cover image when the secret image and the cover image have the same data volume.

\section{Proposed Methodology}

We developed a novel method based on color Transformations between blocks. In the initial part of the planned technique, every tile image $\mathrm{T}$ within the given secret image is match to a target block B in a preselected target image. Since the colour characteristics of $\mathrm{T}$ and $\mathrm{B}$ are totally different from one another, the way to amendment their colour distributions to form them look alike is the main issue here. Color transfer theme in this face $t$ that converts the colour characteristic of an image to be that of another within the 1 $\alpha \beta$ colour area. This idea is a solution to the difficulty and is adopted during this paper, except that the RGB colour area rather than the $1 \alpha \beta$ one is employed to reduce the amount of the desired data for recovery of the original secret image. More specifically, let A and $\mathrm{B}$ be described as 2 pixel sets $\left\{\mathrm{p} 1, \mathrm{p} 2, \mathrm{p} 3, \ldots \ldots \ldots p_{n}\right\}$ and $\left\{p_{1}, p_{2}, \ldots \ldots p_{n}^{*}\right\}$ severally.

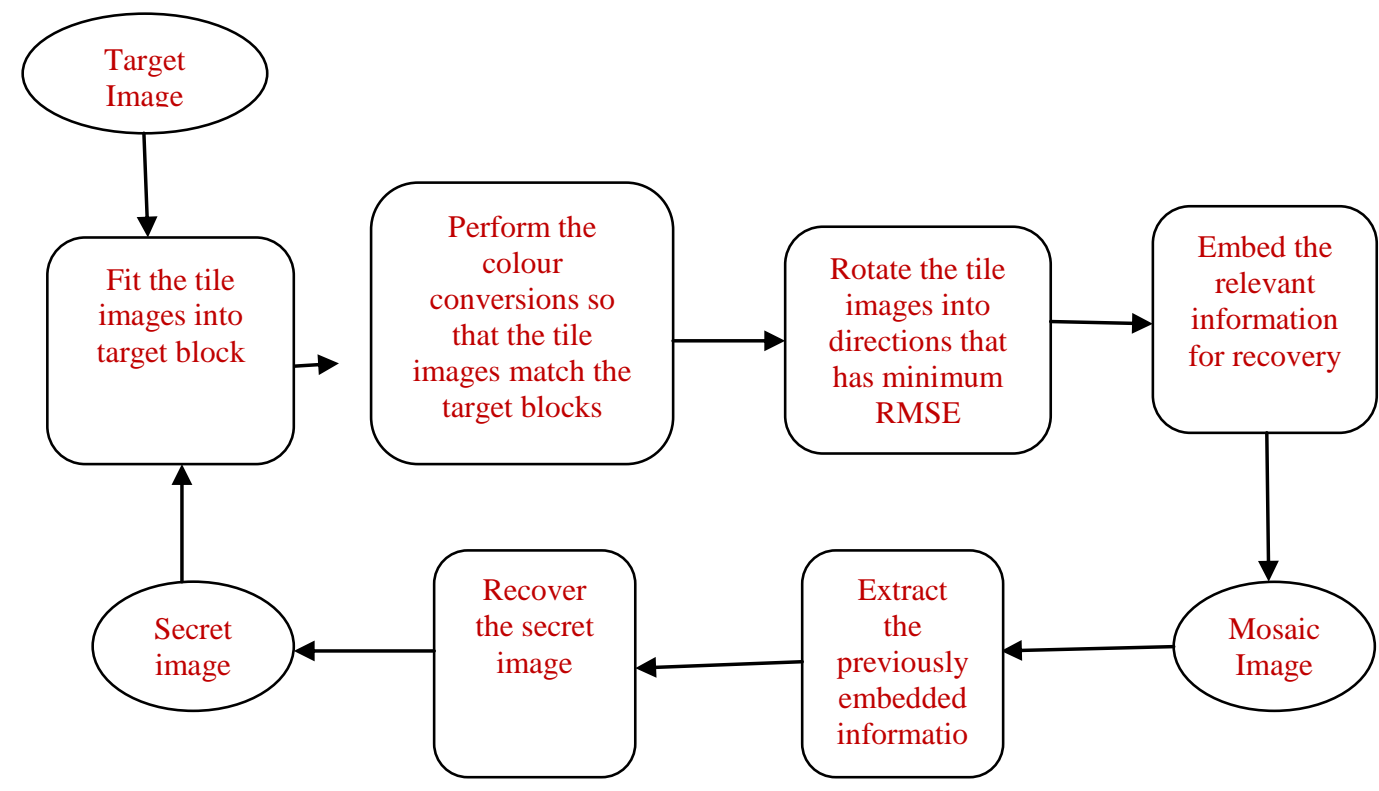

Figure 1. Block Diagram of the Proposed Methodology

\section{1. Mathematical Basics for Proposed Work}

Let the color of every pi be denoted by $\left(r_{i}, g_{i_{3}} b_{i}\right)$. which of every $p^{\prime}{ }_{i}$ by $\left(r_{i}^{*}, g_{i}^{*}, b_{i}^{j}\right)$. At first, we tend to work out the means and standard deviations of $\mathrm{T}$ and $\mathrm{B}$, severally in every of the 3 colour channels R, G, and B by the subsequent formulas:

$$
\mu_{C}=\frac{1}{n} \sum_{i=1}^{n} c i
$$




$$
\begin{aligned}
& \mu_{c}^{\prime}=\frac{1}{n} \sum_{i=1}^{n} c_{i}^{\prime} \ldots \ldots \ldots \ldots \ldots . . . \ldots . . \\
& \sigma_{c}=\sqrt{\frac{1}{n} \sum_{i=1}^{n}\left(c_{i-} \mu_{c}\right)^{2}} \\
& \sigma_{c}^{\sigma}=\sqrt{\frac{1}{n} \sum_{i=1}^{n}\left(c_{i}^{v}-\mu_{c}^{v}\right)^{2}} .
\end{aligned}
$$

where $c_{i}$ and $c_{i}^{*}$, denote the C-channel values of pixels $p_{i}$ and $p_{i}^{*}$ severally, with $\mathrm{C}=\mathrm{R}, \mathrm{G}$,or B. Next, we have a tendency to compute new color values $\left(r_{i}^{p}, g_{i}^{p} b_{i}^{\prime}\right)$ for each $p_{i}$ in $\mathrm{T}$ by

$$
c_{i}^{\prime \prime}=q_{c}\left(c_{i}-\mu_{c}\right)+\mu_{c}^{\prime}
$$

Where $q_{c}=\sigma_{c} / \sigma_{c}^{\sigma}$ is the standard deviation quotient and $\mathrm{c}=(\mathrm{r}, \mathrm{g}, \mathrm{or} \mathrm{b})$. It can be verified easily that the new colour mean and variance of the resulting tile image $T^{\circ}$ are equal to those of $\mathrm{B}$, respectively. To compute the original colour values $\left(\left(r_{i}, g_{i}, b_{i}\right)\right.$ of $p_{i}$ from the new ones $\left(r_{i}^{p}, g_{i_{s}}^{p} b_{i}^{p}\right)$, we use the following formula which is the inverse of (3)

$$
c_{i}=\frac{1}{q_{c}}\left(c_{i}^{\prime \prime}-\mu_{c}^{\prime}\right)+\mu_{c}
$$

Furthermore, we've to bid into the created mosaic image sufficient data concerning the new tile image $T^{*}$ for use within the later stage of convalescent the initial secret image. For this, theoretically we will use (4) to compute the initial pixel price of $p_{i}$. However, the concerned mean and normal deviation values within the formula area unit are all real numbers, and it is impractical to embed real numbers, every with several digits, in the generated mosaic image. Therefore, we limit the numbers of bits we want to represent the relevant parameter values in (3) and (4). Specifically, for every color channel we tend to enable each of the means of $\mathrm{T}$ and $\mathrm{B}$ to have 8 bits with its worth within the vary of 0 to 255 , and also the standard deviation quotient $\mathrm{q}_{\mathrm{c}}$ in (3) to have seven bits with its worth within the vary of 0.1 to 12.8 . That is, each mean is modified to be the closest value within the range of 0 to 255 , and each $\mathrm{q}_{\mathrm{c}}$ is modified to be the closest value in the range of 0.1 to 12.8 . We don't enable $\mathrm{q}_{\mathrm{c}}$ to be zero 0 otherwise the first picture element worth cannot be recovered back by (4) for the reason that $1 / \mathrm{q}_{\mathrm{c}}$ in (4) isn't outlined once $\mathrm{q}_{\mathrm{c}}=0$.

\subsection{Choosing Appropriate Target Blocks and Rotating Blocks to Fit Better with Smaller RMSE Value}

In transforming the color characteristic of a tile image $\mathrm{T}$ to be that of a corresponding target block B as represented higher, how to choose an appropriate B for every $\mathrm{T}$ is a problem. For this, we use the standard deviation of the colors within the block as a live to pick out the foremost similar for each T. Specially; we type all the tile pictures to make a sequence of the tiles $s_{\text {tile }}$ and every of the target blocks to make another, $s_{t \arg e t}$, consistent with the typical values of the standard deviations of the 3 colour channels. Then, we work the primary in $s_{\text {tile }}$ into the primary in $s_{t \text { arg } e t}$, fit the second in S tile into the second in $\mathrm{S}$ target, and so on. 
In addition, after a target block $\mathrm{B}$ is chosen to fit a tile image $\mathrm{T}$ and after the colour characteristic of $\mathrm{T}$ is transformed, we conduct a further improvement on the colour similarity between the resulting tile image $T^{\prime}$ and the target block B by rotating $T^{\prime}$ into one of the four directions $0^{\circ}, 90^{\circ}, 180^{\circ}, 270^{\circ}$ which yields a rotated version of $T^{\prime}$ with the minimum root mean square error (RMSE) value with respect to $\mathrm{B}$ among the four directions for final use to fit $\mathrm{T}$ into $\mathrm{B}$.

\subsection{Embedding Information for Secret Image Recovery}

In order to recover the secret image from the mosaic image, we have to embed relevant recovery information into the mosaic image. In order to recover the secret image from the mosaic image, we've to bid relevant recovery data into the mosaic image. For this, we adopt a way planned by Coltuc and Chassery and apply it to least vital bits of the pixels within the created mosaic image to conduct information embedding. Not like the classical LSB replacement strategies, that substitute LSBs with message bits directly, the reversible distinction mapping technique applies simple integer transformations to pairs of pixel values. Specifically, the method conducts forward and backward integer transformations as follows, respectively, in which (x, y) are a pair of pixel values and (x', $\left.\mathrm{y}^{\prime}\right)$ are the transformed ones.

The method yields high data embedding capacities close to the highest bit rates and has the lowest complexity reported so far. The information required to recover a tile image $\mathrm{T}$ which is mapped to a target block B includes: 1) the index of B;2) the optimal rotation angle of $\mathrm{T} ; 3$ ) the truncated means of $\mathrm{T}$ and Band the standard deviation quotients, of all colour channels; and 4) the overflow/underflow residuals. These data items for recovering a tile image $\mathrm{T}$ are integrated as a four-component bit stream and computed by $\mathrm{M}=[\log$ $\left.[(\mathrm{Ws} \times \mathrm{Hs})] / N_{T}\right]$

BPO in the figure is Bit Plane Order. The two buffers and the bit plane encoder are used to store the image for reference. The image can be extracted whenever the necessity arises. Among 8-bit and 16-bit, the 8-bit was selected for our applications. The output of the encoder used for the decoding process is as usual same in the reconstruction process. The generated alphabet is stored and transmitted to the receiving section for the decoding process without any interruption. In many of the real time application images, the low frequency components consist of more information.

\section{Algorithmic Flow}

In this proposed method it contains mainly two phases. They are one is mosaic image creation and second one is secret image recovery process.

\section{PHASE 1}

Stage 1. Fitting the tile images into the target blocks.

Step 1: Here first we need to compare the sizes of the target and secret image sizes. If they are not equal then we need to resize and equalize them and then divide the secret image into tile images $\left\{T_{1}, T_{2}, T_{3} \ldots \ldots T_{n}\right\}$ and also the target image as $\left\{B_{1}, B_{2} B_{3} \ldots \ldots \ldots B_{N}\right\}$

Step 2: Then calculate both the mean and standard deviation from the above equations (3)and(4), for each tile image $T_{i}$ and target image $B_{j}$ respectively for i,j=1 ...

Step 3: Now we have the set of tile images as $S_{\text {title }}=\left\{T_{1}, T_{2}, T_{3} \ldots \ldots \ldots T_{n}\right\}$ and target blocks are $S_{\text {target }}=\left\{B_{1}, B_{2} B_{3} \ldots \ldots \ldots B_{N}\right\}$ then by sorting them according to the mean and standard deviation values we need to map the tile image set to the target blocks in 1-to-1 manner then resulting mapping sequence $\mathrm{L}$. 
Step 4: So create the mosaic image $\mathrm{F}$ by fitting the tile images into the corresponding blocks according to $\mathrm{L}$.

Stage 2: Rotating Images

Step 1: For each colored transformed tile image $T_{1}$ calculate the RMSE values in F with respected to corresponding target block $B_{J i}$ after rotating $T_{i}$ into directions of $\theta=0,90,180,270,360$ respectively.

PHASE 2: secret image retrieval

Stage1: Extracting the secret image recovery information.

Step 1: Extract the bit stream I from the F by reversion scheme and decode them to get the below items1) the number of iterations $N_{i}$ for embedding $M_{i}^{\sigma}$ the total number of used pixel pairs $N_{\text {pair }}$ in the last iteration.

Step 2: Repeat the above step to extract the $M_{i}^{\sigma}$.

Step 3: Then decrypt the bit stream $M_{i}^{x}$ into $M_{i}$ by the using of key K.

Step 4: Decompose $M_{t}$ into $\mathrm{n}$ bit streams $M_{1}$ through $M_{n}$ for the $\mathrm{n}$ to-be-constructed tile images $T_{1}$ through $T_{n}$ in $\mathrm{S}$, respectively.

Step 5: Decode $M_{i}$ for each tile image $T_{1}$ to obtain the following data items: 1) the index $\mathrm{j}_{\mathrm{i}}$ of the block $B_{i j}$ in F corresponding to $T_{i} 2$ ) the optimal rotation angle $\theta^{\circ}$ of $T_{i} ; 3$ ) the means of $T_{i}$ and $B_{i j}$ and the related standard deviation quotients of all color channels.

\section{Stage 2: Recover the secret image}

Step 6: Extract one by one in a raster-scan order the tile images $T_{i}, \mathrm{i}=1$ through $\mathrm{n}$, of the desired secret image $S$ by the following steps: 1) rotate in the reverse direction the block through the optimal angle2) use the extracted means and related standard deviation quotients to recover the original pixel values in $T_{i}$ according to (4); 3) take the outputs as the final pixel values, resulting in a final tile image $T_{i}$.

Step 7: Combine all the final tile images to get desired secret image $\mathrm{T}$.

\section{Simulation Results}

The simulation results of the images using MATLAB are included in the preceding section.

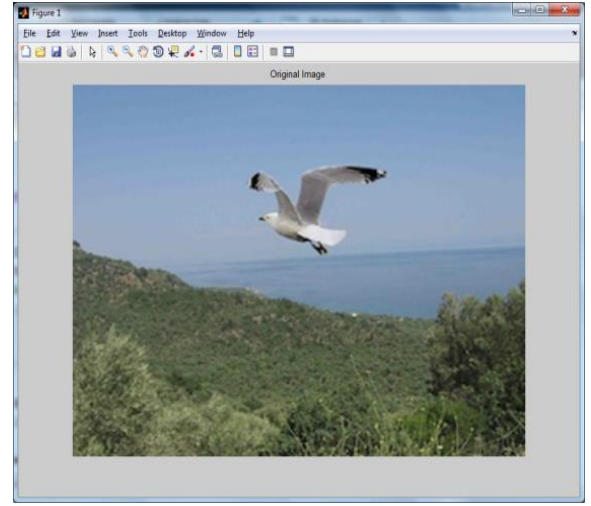

Figure 1. Original Image

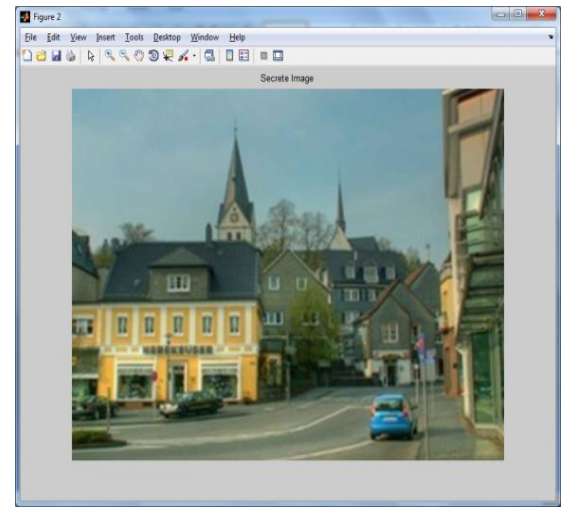

Figure 2. Secret Image 


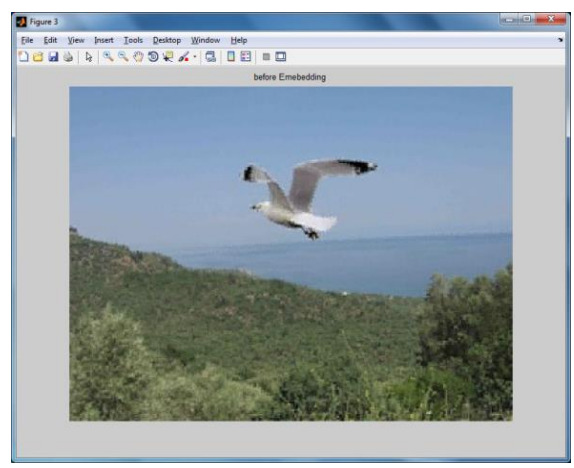

Figure 3. Before Embedding Mosaic Image

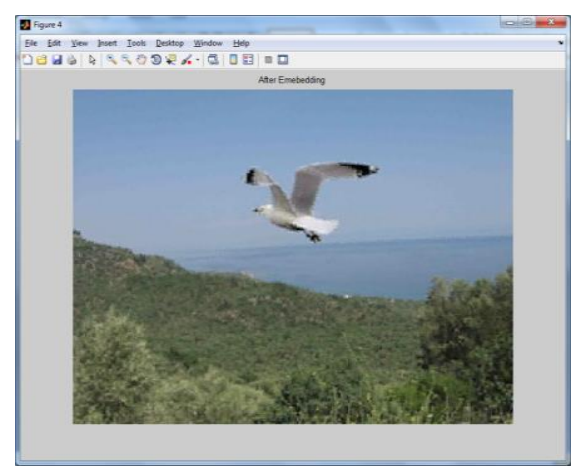

Figure 4. After Embedding Mosaic Image

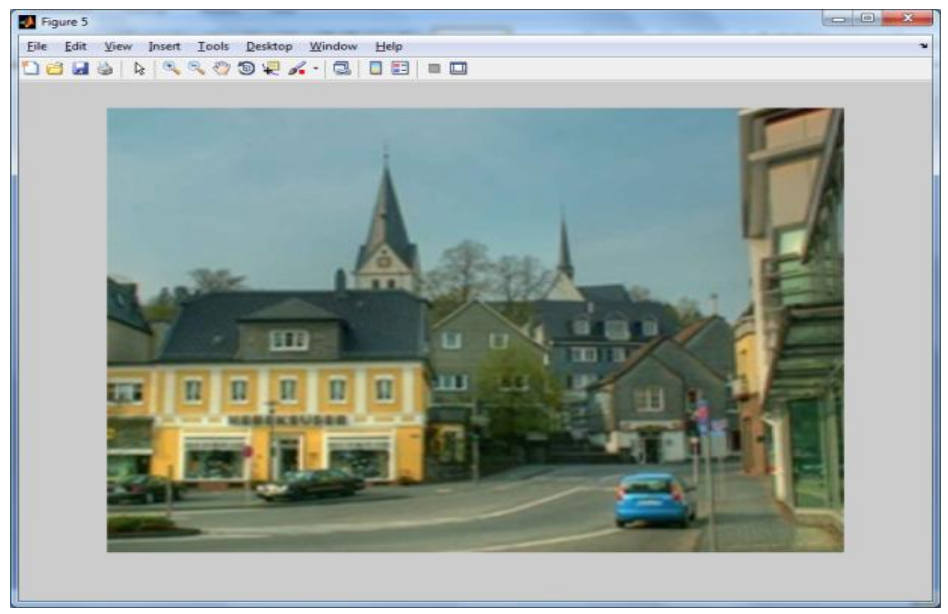

Figure 5. Extracted Image

We observed the following graphs for various sizes and various parameters.

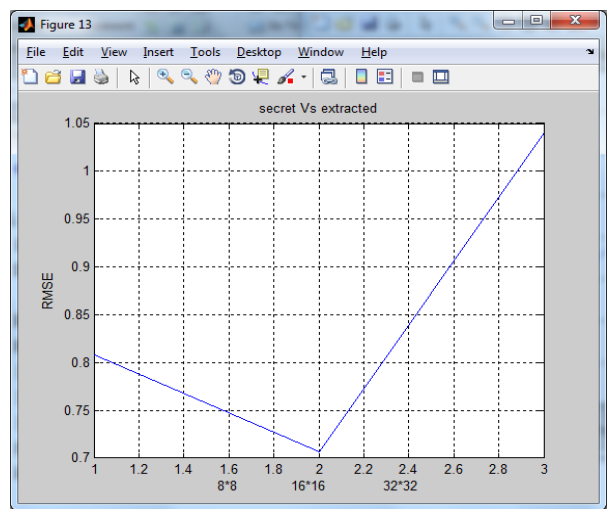

Figure 6. Graph Showing Secret vs. Extracted (RMSE)

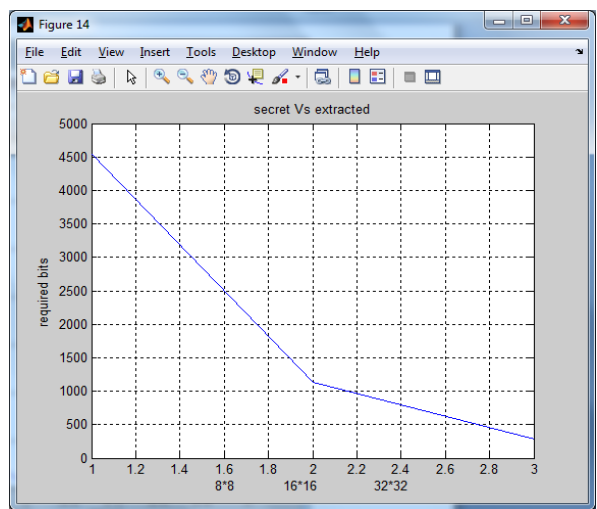

Figure 7. Graph Showing Secret vs. Extracted (Required bits) 


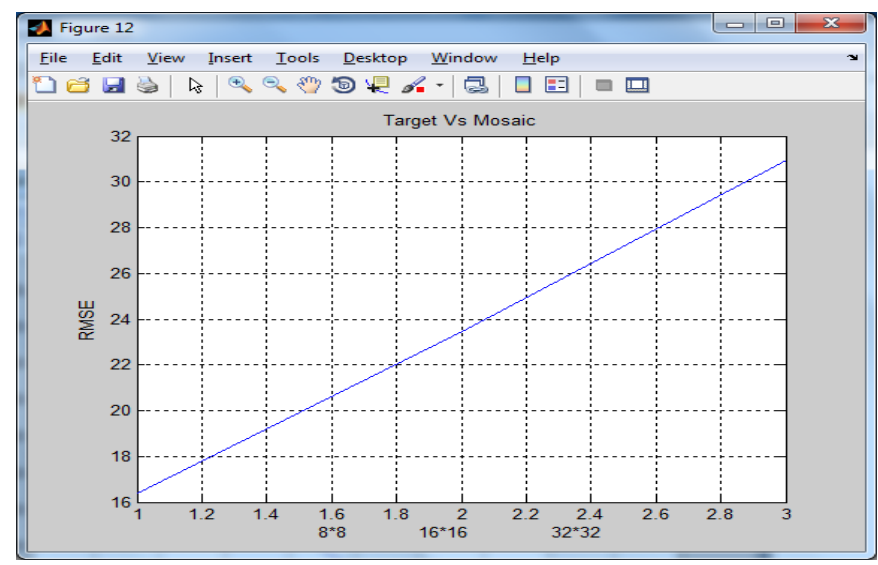

Figure 8. Graph Showing Target vs Mosaic (RMSE)

This Method has been applied to various sizes like $8 * 8,16 * 16,32 * 32$ and their outputs are shown below.

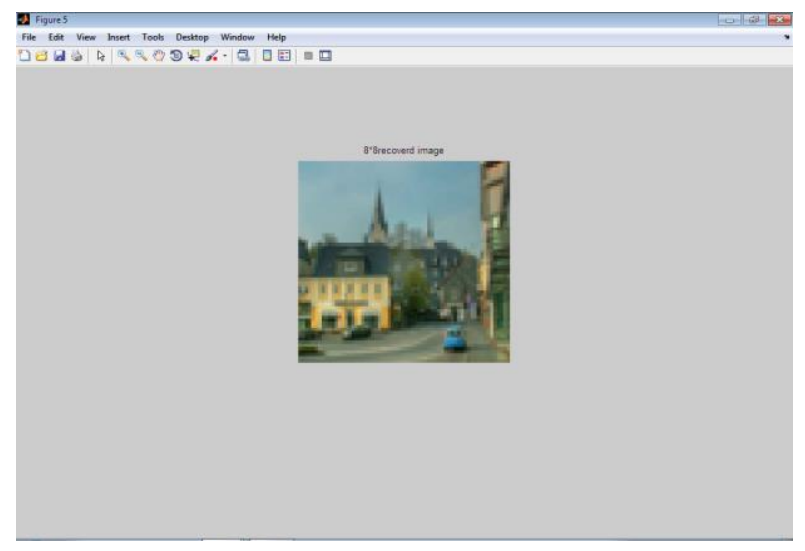

Figure 9. Recovered Image of Size $8 * 8$ Block

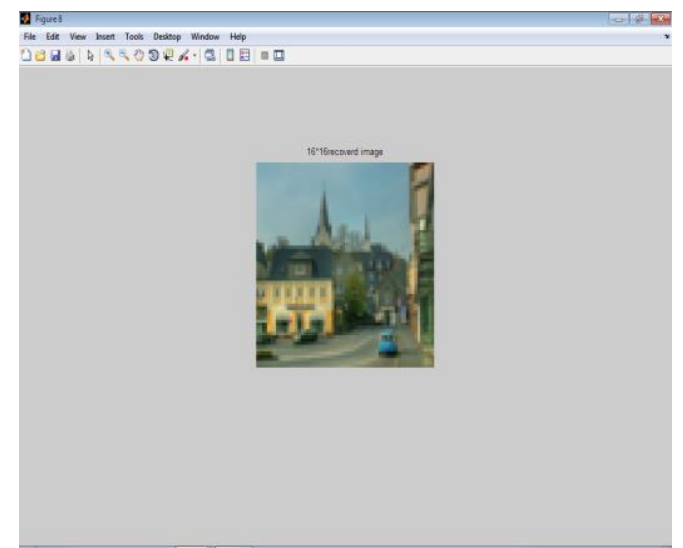

Figure 10. Recovered Image of Size $16 * 16$ block

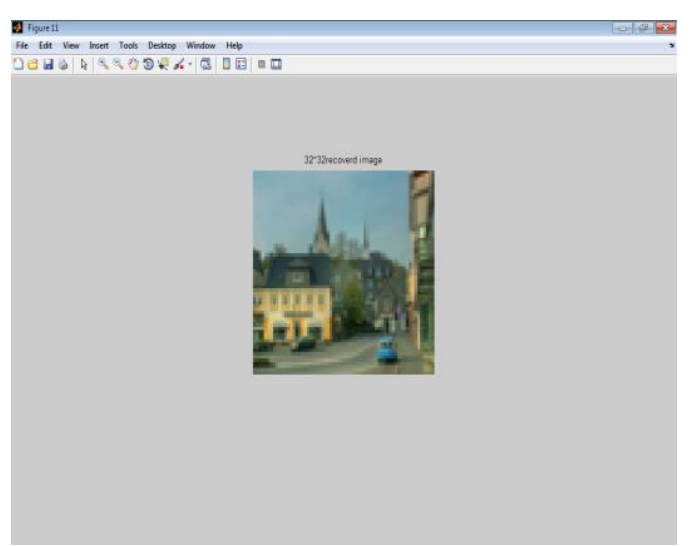

Figure 11.Recovered image of Size $32 * 32$ block

By using these we have tabulated different parameters like RMSE, PSNR, run time, required bits are shown in Table 1. 
Table 1. Various Measured Parameters for Different Sizes of Image Blocks

\begin{tabular}{|l|c|c|c|}
\hline \multicolumn{1}{|c|}{ Parameters } & $\mathbf{8}^{\star} \mathbf{8}$ & $\mathbf{1 6} \mathbf{1}^{*} \mathbf{6}$ & $\mathbf{3 2} \mathbf{3 2}$ \\
\hline $\begin{array}{l}\text { Root Mean Square Error } \\
\text { (Target vs. Secret) }\end{array}$ & 16.39 & 23.94 & 30.93 \\
\hline $\begin{array}{l}\text { Root Mean Square Error } \\
\text { (Secret vs. Extracted) }\end{array}$ & 0.81 & 0.71 & 1.04 \\
\hline $\begin{array}{l}\text { Required Bits } \\
\text { Peak Signal to Noise Ratio (dB) }\end{array}$ & 4544 & 1136 & 284 \\
\hline Run Time (seconds) & 40.01 & 51.18 & 47.82 \\
\hline
\end{tabular}

\section{Conclusion}

Hiding the data in digital images has been area of interest in the digital image processing domain. Although so much work has been carried out in the literature to resolve the issues like increasing the data capacity, creating the secret image alike of target image but most of the works fails to meet the practical requirements. This paper presents an approach where mosaic image generation has done by dividing the secret image into fragments and transforming their respective colour characteristics into corresponding blocks of the target image. Usage of the Pixel colour transformations helps to yield the lossless recovered image based on the untransformed colour space values. Generation of the key plays an important role to recover the data from the secret image in lossless manner. Finally the same approach can be performed on videos also which helps to eliminate the flickering artifact to achieve the lossless data recovery in motion related videos. The experimental results shows good robust behavior against all incidental and accidental attacks and compare to the conventional algorithms performance evaluation has been increased in a significant way.

\section{Future Scope}

The proposed method has been written on the digital images, in this work images are used as media to hide the secret image by using the an approach where mosaic image generation has done by dividing the secret image into fragments and transforming their respective colour characteristics into corresponding blocks of the target image. Usage of the Pixel colour transformations helps to yield the lossless recovered image based on the untransformed colour space values. So in extension work we did the same algorithm on the digital videos. The approach towards videos is totally different from the images, so algorithm on videos is the future expansion for our contribution. 


\section{Acknowledgements}

I feel profoundly indebted to my guide Dr. Shaik. Mahaboob Basha for the opportunity to do the work under his guidance. It gives pleasure in completing the work by his timely advices.

\section{References}

[1] J. Fridrich, "Symmetric ciphers based on two-dimensional chaotic maps," Int. J. Bifurcat. Chaos, vol. 8, no. 6, (1998), pp. 1259-1284.

[2] G. Chen,Y. Mao, and C. K. Chui, "A symmetric image encryption scheme based on 3D chaotic cat maps," Chaos Solit. Fract., vol. 21, no. 3, (2004), pp. 749-761.

[3] L. H. Zhang, X. F. Liao, and X. B. Wang, "An image encryption approach based on chaotic maps," Chaos Solit. Fract., vol. 24, no. 3, (2005), pp. 759-765.

[4] H. S. Kwok and W. K. S. Tang, "A fast image encryption system based on chaotic maps with finite precision representation," Chaos Solit. Fract., vol. 32, no. 4, (2007), pp. 1518-1529.

[5] S. M. Basha and B. C. Jinaga, "A New Approach Based on Order Reduction using sub image formation in minimizing the Computation time for Image Compression", International Journal of Signal Processing, Image Processing and Pattern Recognition, vol. 8, no. 3, (2015).0

[6] S. M. Basha and B. C. Jinaga, "Wireless Motor Controller over Bluetooth Network", IPASJ International Journal of Computer Science, (IIJCS), vol. 2, no. 10, (2014) October.

[7] S. M. Basha and B. C. Jinaga, "Modified Robust Technique for Intra and Inter Constraint based on Video Enhancement using Colour Modeling of Countable Frames International Journal of Signal Processing", Image Processing and Pattern Recognition, vol. 7, no. 4, (2014), pp. 399-4089.

[8] S. M. Basha and B. C. Jinaga, "A Novel Optimized Golomb-Rice Technique for the Reconstruction in Lossless Compression of Digital Images”, ISRN Signal Processing, vol. 2013, (2013).

[9] S. M. Basha and B. C. Jinaga, "A Novel Optimum Technique for JPEG 2000 Post Compression Rate Distortion Algorithm", ACEEE International Journal of Information Technology, vol.1, no. 2, (2011), pp. $41-46$.

[10] S. M. Basha and B. C. Jinaga, "Robust Reconstruction of the Image in JPEG2000 compression by quaternion wavelet transform", Journal of Instrumentation Society of India of ,Indian Institute of Science, Bangalore in June, (2012).

[11] S. M. Basha and B. C. Jinaga, "A robust approach for qualitative compression in JPEG 2000 standard", IEEE Prime Asia 2012 International Conference held on at BITS, Hyderabad (2012).

[12] S. M. Basha and B. C. Jinaga, "A novel response dependent image compression algorithm to in Reduce the Non-linear effects in colour images using JPEG", IEEE International Conference (SIBIRCON2010) July, (2010).

[13] S. Behnia, A. Akhshani, H. Mahmodi, and A. Akhavan, "A novel algorithm for image encryption based on mixture of chaotic maps," Chaos Solit. Fract., vol. 35, no. 2, (2008), pp. 408-419.

[14] D. Xiao, X. Liao, and P. Wei, "Analysis and improvement of a chaos based image encryption algorithm," Chaos Solit. Fract., vol. 40, no. 5, (2009), pp. 2191-2199.

[15] V. Patidar, N. K. Pareek, G. Purohit, and K. K. Sud, "A robust and secure chaotic standard map based pseudorandom permutation substitution scheme for image encryption," Opt. Commun., vol. 284, no. 19, (2011), pp. 4331-4339.

[16] S. M. Basha and B. C. Jinaga, "A Robust Image Compression Algorithm using JPEG 2000 Standard with Golomb Rice Coding", International Journal of Computer Science and Network Security, vol. 10, no. 12, (2010), pp. 26-33.

\section{Authors}

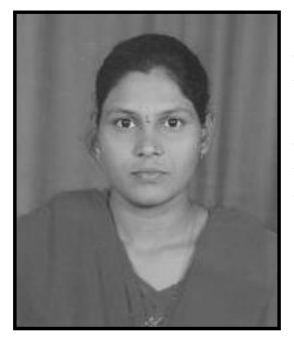

Tummala. Lakshmi Prasanthi, she is currently is pursuing his M.Tech specialization in Digital Electronics and Communication Systems in Priyadarshini College of Engineering and Technology, Nellore which is affiliated to JNTUA. She is doing her research work under the guidance of Shaik. Mahaboob Basha. 


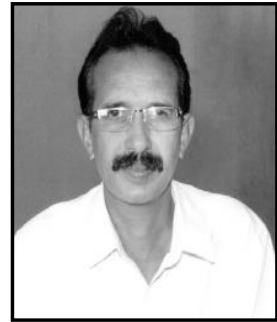

Shaik. Mahaboob Basha, he is working as Professor and Head of the Department of Electronics and Communication Engineering in Priyadarshini College of Engineering and Technology, Nellore. He has done his $\mathrm{PhD}$ from J.N.T.University, Hyderabad.He is the member of Engineering Professional bodies like IEEE, IE(I),ACM and ISOI . He has published 15 papers in reputed International and National journals and conferences.

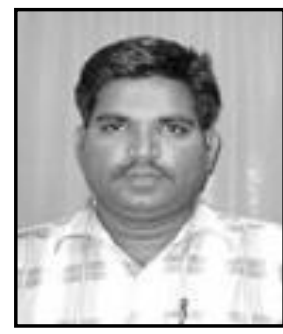

Jogi Srinivasulu, he is working as Assistant professor of the Department of Electronics and Communication Engineering in Priyadarshini College of Engineering and Technology, Nellore. His teaching experience is 7 years. He qualified NET june-2013 and he has also qualified GATE march-2015. 
International Journal of Signal Processing, Image Processing and Pattern Recognition Vol.8, No.10 (2015) 